\title{
Using multielectrode arrays to investigate neurodegenerative effects of the amyloid- beta peptide
}

\author{
Steven Schulte ${ }^{1}$, Manuela Gries ${ }^{1}$, Anne Christmann ${ }^{1}$ and Karl-Herbert Schäfer ${ }^{1,2^{*}}$
}

\begin{abstract}
Background: Multielectrode arrays are widely used to analyze the effects of potentially toxic compounds, as well as to evaluate neuroprotective agents upon the activity of neural networks in short- and long-term cultures. Multielectrode arrays provide a way of non-destructive analysis of spontaneous and evoked neuronal activity, allowing to model neurodegenerative diseases in vitro. Here, we provide an overview on how these devices are currently used in research on the amyloid- $\beta$ peptide and its role in Alzheimer's disease, the most common neurodegenerative disorder.

Main body:: Most of the studies analysed here indicate fast responses of neuronal cultures towards aggregated forms of amyloid- $\beta$, leading to increases of spike frequency and impairments of long-term potentiation. This in turn suggests that this peptide might play a crucial role in causing the typical neuronal dysfunction observed in patients with Alzheimer's disease.

Conclusions: Although the number of studies using multielectrode arrays to examine the effect of the amyloid- $\beta$ peptide onto neural cultures or whole compartments is currently limited, they still show how this technique can be used to not only investigate the interneuronal communication in neural networks, but also making it possible to examine the effects onto synaptic currents. This makes multielectrode arrays a powerful tool in future research on neurodegenerative diseases.
\end{abstract}

Keywords: Neurodegenerative diseases, Alzheimer's disease, Multielectrode arrays, Pharmacology, Target identification, Drug discovery, Neural circuit activity

\section{Background}

Neurodegenerative diseases are hallmarked by a massive, pathological death of neurons, which leads to a decline in cognitive and/or motoric abilities. Symptoms depend on the neural compartment that is impacted and its function. The most common neurodegenerative disorder, Alzheimer's disease (AD), is characterized by a loss of neurons in certain cerebral cortical regions, including hippocampus and temporoparietal cortex (St

\footnotetext{
* Correspondence: karlherbert.schaefer@hs-kl.de

'Department of Informatics and Microsystems and Technology, University of Applied Science Kaiserslautern, 66482 Zweibrücken, Germany

${ }^{2}$ Department of Pediatric Surgery, Medical Faculty Mannheim, University of Heidelberg, 68167 Mannheim, Germany
}

George-Hyslop and Petit 2005), followed by cognitive disturbances. Based on previous studies to develop potential treatments of neurodegenerative diseases and the etiological mechanisms involved, it has become a crucial task to understand the role of possibly harmful peptides in processes leading to neuronal cell death. Given the electrogenic features of neural tissue, studying the impact of such endogenous neurotoxic substances on neuronal function is an absolute demanding goal. So far, investigations on the impact of these molecules have been based largely on electrophysiological experiments. Loss of electrical functionality indicates an impairment of compromised neurons and is seen as the initiation point of the typical symptoms in neurodegenerative

(c) The Author(s). 2021 Open Access This article is licensed under a Creative Commons Attribution 4.0 International License, which permits use, sharing, adaptation, distribution and reproduction in any medium or format, as long as you give appropriate credit to the original author(s) and the source, provide a link to the Creative Commons licence, and indicate if changes were made. The images or other third party material in this article are included in the article's Creative Commons licence, unless indicated otherwise in a credit line to the material. If material is not included in the article's Creative Commons licence and your intended use is not permitted by statutory regulation or exceeds the permitted use, you will need to obtain permission directly from the copyright holder. To view a copy of this licence, visit http://creativecommons.org/licenses/by/4.0/.
} 
disorders. To reveal the influence of potentially neuropathogenic peptides such as amyloid- $\beta(A \beta)$, whose aggregated form might be the culprit in AD-related neurodegeneration, its direct effect upon single neurons as well as on whole neural networks needs to be evaluated. For these purposes, different methods have emerged during the last decades and to choose an appropriate one largely depends on the scope of analysis. For single-cell examination, the patch clamp technique is a widely used tool, allowing to record intracellular voltages of single neurons using sharp glass pipettes, which restricts measurements to a few neurons per experiment. Although multi-cell approaches have been developed (Wagner et al. 2015), these recordings can be time and effort consuming.

To deduct signals of multiple neurons or whole neural networks simultaneously, multielectrode arrays (MEAs) have been used for many decades. MEAs allow to investigate the impact of neurotoxic compounds upon complex neuronal networks in vitro and in specific cases even in vivo (Wood et al. 2004). The MEA technology is based on extracellular recordings and on the performance of mainly metal electrodes which are arranged in large arrays. In contrast to patch clamp techniques, these devices provide a non-invasive method to record electrical signals from whole neuronal networks and, by increasing the number of electrodes in the array, allow to magnify the scope to single neurons. Moreover, individual approaches also allow to stimulate the networks with one of the recording electrodes.

Although there are several other electrophysiological methods which can be used to elucidate the effect of pharmacologically active substances onto neural networks in vitro, like calcium imaging, MEA technique has some key features reviewed here that make it a powerful tool in the analysis of neurodegenerative diseases. This review tries to provide a short overview of the potential use of MEAs in the evaluation of neuropathological effects of $A \beta$. First, there will be a short description of MEA technology and its basics followed by a state of the art of AD research using the MEA technology. Finally, current work in the field of AD research using MEAs is presented.

\section{Main text}

\section{MEA technology and applications}

MEAs are substrate-integrated arrays of ten to thousands of metal electrode contacts (Fig. $1 \mathrm{~A}$ and B) and offer the possibility to record neuronal activity in vitro (Fig. $1 \mathrm{C}$ ) as well as in vivo, non-invasively and with high spatial and temporal resolution (Ness et al. 2015). The number of electrodes is a key feature enhancing spatial resolution in particular, revealing the main advantage over e.g. EEG recordings (Obien et al. 2015). The shape of the electrodes can vary from planar (Fig. 1D) to 3dimensional (Fig. 1E and F; (Decker et al. 2019)) and even mimicking the structure of the natural anchor proteins of the extracellular matrix, such as collagen, to improve cell coupling and adhesion (Nowduri et al. 2020). The MEA technique allows to record local field potentials (LFP) and extracellular action potentials (EAPs) simultaneously. In specific cases, using the 3D electrode in vitro approach, a deduction of subcellular activity (e.g. from dendrites, somata and axons) can be accomplished (Spira and Hai 2013). Moreover, long-term experiments that include the implementation of convective perfusion for automated culturing of organotypic slices, open interesting perspectives to follow the impact of individual compounds along prolonged time axes (Killian et al. 2016). EAPs are defined as action potentials recorded by electrodes placed in the extracellular space, in contrast to intracellular action potentials (IAPs) obtained in patch clamp measurements. Neuronal EAPs usually are around tens to hundreds of microvolts in amplitude and less than 2 milliseconds (ms) in duration (Buzsáki et al. 2012). MEA microelectrodes detect the changes in the extracellular field which are caused by current flows of ionic processes of the neurons closest to the electrode, while this is not restricted to neuronal cells (Quiroga et al. 2013). This electrical field is usually referred to as the LFP and resembles a superposition of all ionic processes (Herreras 2016). Thus, any neuronal transmembrane current contributes to the potential detected on a microelectrode, while the characteristics of this potential resemble the sum of every neuronal source in the vicinity of this particular electrode (Buzsáki et al. 2012). Hence, the EAP of many neurons can be the source of the LFP, though it is still unclear how EAPs contribute to the extracellular field in detail (Obien et al. 2015).

Action potentials measured at an electrode are commonly referred to as "spikes", which in the context of extracellular recordings are characterized as voltage signals that exceed a certain threshold; if several spikes occur in succession with an interspike interval of less than 3ms, they are commonly referred to as "bursts" (Fig. 2 A). These signals exhibit certain features like waveform, amplitude and frequency, all of which depend on neuronal subtype, distance from the electrode and stimulus (Fig. 2B and C). This makes feature extraction and spike sorting an essential part in the analysis of these recordings, to understand which kinds of neurons take part in the communication within a neural network (Lewicki 1998).

This "crosstalk" is often recorded spontaneously, that is, without any application of external stimuli, to create a baseline which can serve as a reference. On the other hand, these responses can also be evoked, either by applying substances with a known or yet to be tested effect 

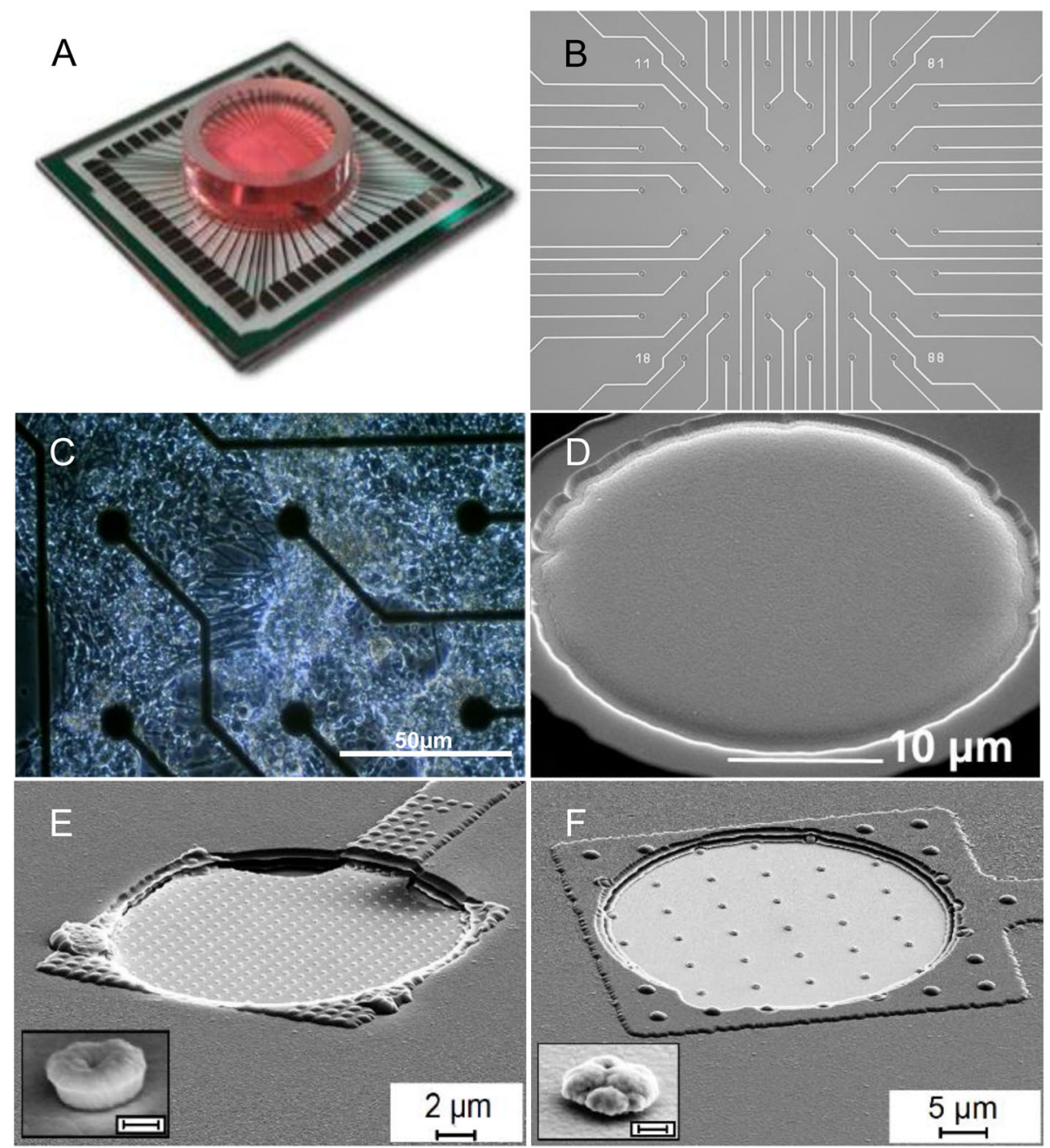

Fig. 1 Exemplary pictures of MEA chips and electrodes of different shapes. A multielectrode array from Multichannel Systems $\odot$ with 60 electrodes and chamber containing cell culture medium. B Arrangement of electrodes in an array of 60 . C Clusters of myenteric neurons growing on planar microelectrodes, scale bar $50 \mu \mathrm{m}$. D SEM picture of a planar electrode, scale bar $10 \mu \mathrm{m}$. E and $\mathbf{F}$ SEM pictures of nanostructured MEA electrodes with tube- and mushroom-like nanostructures, scale bars 2 and $5 \mu \mathrm{m}$, respectively (insets: magnification of nanostructures, scale bar: $200 \mathrm{~nm}$; taken with permission from Decker et al., 2019)

on neural tissue, by electrically stimulating cells through the integrated electrodes (Hales et al. 2012), or a combination of both. In this regard, an interesting approach to understand the ongoing processes within the neuronal networks, or to identify role and type of neurons involved, is the pharmacological blockage of individual subtypes with subsequent analysis of the impact on spike formation (Gramowski et al. 2006).

Taken together, these modifications can bring the experimental environment closer to the in vivo situation, making in vitro MEA recordings a powerful tool in pharmacological and neurophysiological research. Many studies have delivered insight on how to use these devices i.e. for drug-screening, in order to estimate neurotoxicity and overall influence of substances onto isolated compartments of the nervous system (Morefield et al.
2000; Gopal et al. 2011; Gramowski et al. 2011), or even to use neurons as biosensors for a wide range of chemicals using methods of genetical engineering (Patriarchi et al. 2018; Sabatini and Tian 2020).

However, the application of MEAs extends beyond recordings of isolated neural networks in vitro to the implantation of electrodes into cerebral tissue of animals and humans, where again the summation of signals of many sources is recorded. This provides an even closer look into the in vivo situation but also makes analyses like spike sorting a more complex task, due to the drastically increased number of sources that give rise to the signals at a particular electrode. In this context, new approaches of data processing have emerged out of the ongoing progress of artificial intelligence, from methods of Deep Learning in particular. Here, artificial neural 

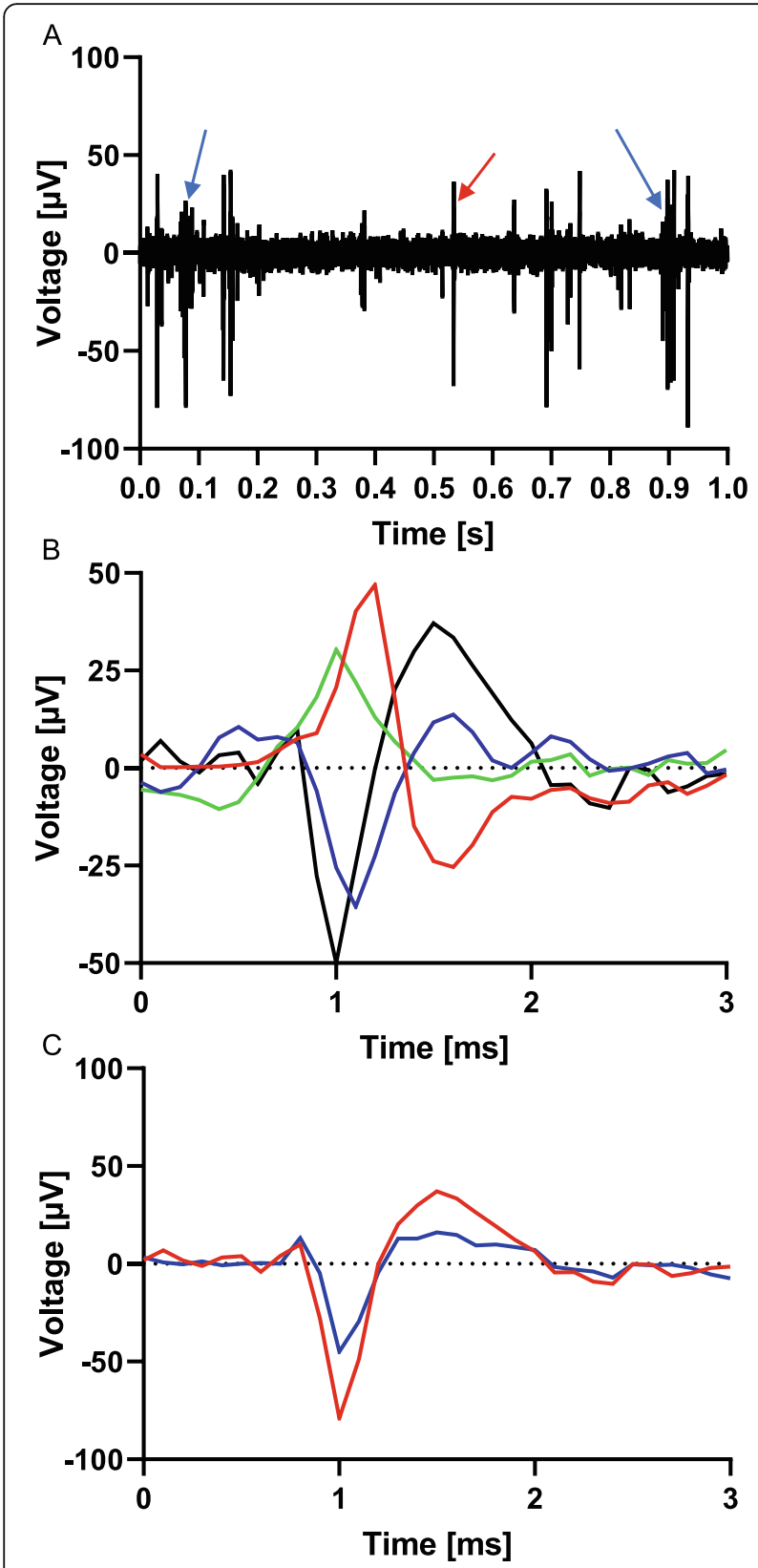

Fig. 2 Illustration of signals recorded with multielectrode arrays. A One second stream of recording from a single electrode, including single spikes (red arrow) and bursts (blue arrows). B Overlay of single spikes of different waveforms, which suggest different neuronal subtypes as the source. c Overlay of two spikes with different amplitudes but similar waveforms, either indicating different neuronal subtypes or distances of the respective spiking neurons from the recording electrode

networks are trained to recognize spikes using large sets of simulated signals (see (Tavanaei et al. 2019) for a review of current concepts). These programs are not only able to detect spikes in an unsupervised fashion, but can also perform tasks like spike sorting (Saif-Ur-Rehman et al. 2019) and predicting the distance of neurons from the recording electrode (Buccino et al. 2018).

Nevertheless, there are several advantages of in vitro MEA recordings of dissociated neurons over in vivo approaches and acute brain slice recordings, the most pragmatic being the fact that cultures and recordings can be sustained for a prolonged period (Potter and Demarse 2001). This in turn opens up the opportunity to study the formation and development of the geometry and electrical activity of neural networks (Shahaf and Marom 2001; Hofmann and Bading 2006). In addition, temporal resolution of in vitro MEA recordings can outrun techniques for imaging neuroelectrical activity, like 2-photon calcium imaging (Delgado Ruz and Schultz 2014). Considering these advantages, together with the possibility to study the effect of pharmacologically active peptides, recordings of cultured neurons on MEAs may facilitate the study of neurodegenerative disorders like $\mathrm{AD}$ (Jones et al. 2011).

\section{Alzheimer's disease}

$\mathrm{AD}$ is mainly characterized by a loss of neurons in distinct areas of the brain (i.e., hippocampus and temporoparietal neocortices). Most research on therapeutics has focused on the histopathological hallmarks, cortical amyloid plaques and neurofibrillary tangles, in association with their most insoluble components, the $\mathrm{A} \beta$ peptide (Glenner, G. and Wong 1984) and the microtubuleassociated protein tau (Wood et al. 1986), respectively. In this context, aggregates termed senile or amyloid plaques are often seen as the culprit in AD pathophysiology and are another prominent neuropathological feature. These aggregates are complex, extracellular, fibrillar deposits of several proteins, while the main component is the neurotoxic $A \beta_{42}$ peptide, a 42 amino acids long molecule derived from the $\beta$-amyloid precursor protein $\beta$ APP (St George-Hyslop and Petit 2005). In this review, we will focus on studies that analyzed the effect of $A \beta$ on to neural networks using in vitro MEA approaches, as different isoforms of $A \beta$ are present in all of the plaques that are linked to 'normal' aging and Alzheimer's disease, regardless of size, shape, aggregation state, location, or overall composition (Walker 2020).

There are several propositions that have been postulated to explain cognitive dysfunctions accompanying this neurodegenerative disease on the neurophysiological level. The current understanding is that these damages are most probably not directly originated in neuronal apoptosis, at least not in earlier stages of $\mathrm{AD}$. $\mathrm{A} \beta_{42}$ is known to bind especially to the $\alpha 7$ subunit of the nicotinic acetylcholine receptor (nAChR), at least in the central nervous system (Wang et al. 2000). This might explain why cholinergic neurons seem to be the group of cells that are affected first by the disease. 
Multiple studies have delivered evidence indicating that oligomers of $A \beta$ also have a direct effect on synapses and that the typical symptoms of $\mathrm{AD}$ are derived from an impaired synaptic function (Townsend et al. 2006; H. Alzoubi et al. 2011; Koffie et al. 2011). This also results in changes in synaptic plasticity, a factor commonly described as the ability of neural tissue to form new synaptic connections and enhance existing ones as a part of memory and learning. To investigate on this particular property, long-term potentiation (LTP) is often taken to model synaptic plasticity, and the MEAtechnology is a valuable tool to measure these events, normally with conventional MEAs, but also with especially designed electrode arrays where hippocampal slices can be placed appropriately on the MEA, so that the Schaffer collaterals can easily be addressed (Zheng et al. 2021). LTP can be described as an enhancement of synaptic activity following short, high-frequency stimulation through afferent connections (Chen et al. 2000). This electrical activation, which can be simulated in vitro by applying voltages of certain frequencies via integrated electrodes, can evoke a particular kind of signals known as excitatory post-synaptic potentials (EPSPs), which are enabled through activation of voltage-gated neuronal dendrites (Johnston et al. 1996). A suppression of LTP in hippocampal tissue by monomeric and oligomeric $A \beta_{42}$, respectively, could be found in several studies, suggesting a deleterious impact of $\mathrm{A} \beta_{42}$ on synaptic plasticity (Chen et al. 2000; Wang et al. 2002).

Besides these findings, there seems to be a rise in excitability of neurons in the progress of AD. This hyperexcitability may be linked to changes in the geometry of dendrites, which can lead to alterations of their electrical properties (Spruston 2008). Indeed, reduction of dendritic branching and length are found in hippocampal neurons of patients with AD (Grutzendler et al. 2007), which may render a neuron electrically more compact. This in turn could increase the efficiency with which synaptic currents are translated into postsynaptic and axosomatic depolarization, which would then raise action potential output (Johnston et al. 1996). Another consequence thereof might be abnormal circuit synchronization, which has been found to contribute to cognitive dysfunction in patients with $\mathrm{AD}$ (Minkeviciene et al. 2009).

\section{Revealing amyloid- $\beta$ toxicity in multielectrode array experiments}

During the last decade, several studies have investigated the relationship of aggregated $\mathrm{A} \beta$ onto neural networks grown on MEAs. Most of this research has been concentrated onto hippocampal neurons, as this cerebral area seems to be the first affected by the disease, leading to typical early symptoms of dementia. Cultures are either integrated as whole slices of hippocampal tissue or as dissociated cells. While the number of studies using the MEA technology to examine the impact of monomeric and aggregated species of $A \beta$ onto neural tissue in vitro is comparatively low, they nevertheless show the power of this technique regarding the simulation of the in vivo situation, mimicking the communication and communication deficits between different cerebral compartments by electrical and chemical evocation of interneuronal signaling.

The changes in this communication elicited by $A \beta$ include frequency and amplitude of spikes not only from neuronal, but also synaptic sources. Varghese and coworkers showed significant changes in firing rates of hippocampal neurons in rats following long-term application of different concentrations of oligomeric $A \beta_{42}$ (Varghese et al. 2010) ranging between 100nM and $20 \mu \mathrm{M}$. This study showed a dose-dependent relationship between $A \beta_{42}$ concentration and the duration for reaching total abolishment of spiking activity. While all used concentrations of $A \beta_{42}$ oligomers increased spiking activity over the course of a few hours, firing rates were depleted shortly thereafter. Increasing concentration not only led to earlier attenuation of electrophysiological function, but also to significant neural cell death. Furthermore, this effect could be partially reversed by coculturing hippocampal neurons with the antiamyloidogenic natural compound curcumin, which prevented firing rates from declining. Curcumin is a phenolic yellow pigment derived from the curry spice mixture that has been shown to have potent antiinflammatory and antioxidant activities, and binds to species of $A \beta$ (Yang et al. 2005).

The initial increase of spiking rates found here was also recently observed in another study (Henderson et al. 2019). There, the authors ascribe this feature to a hyperexcitability of hippocampal neurons following the application of $A \beta_{42}$ oligomers over a course of $6 \mathrm{~h}$, which was formerly described in a paper examining an APP mouse model of AD (Šišková et al. 2014).

The neuroprotective abilities of curcumin in the presence of $A \beta_{42}$ oligomers were corroborated by Hoppe et al., which showed that curcumin prevents aggregated species of $A \beta$ from decreasing total LFP of hippocampal neurons in a long-term measurement of $24 \mathrm{~h}$ (Hoppe et al. 2013). The authors propose that curcumin decreases $A \beta_{42}$ induced attenuation of synaptically propagated neuronal activity. Several studies have put the scope of their MEA-based experiments to signals derived from synaptic currents and the effect of $A \beta$ oligomers onto it. As it has been shown in the past, the cognitive dysfunction observed in $\mathrm{AD}$ patients may be caused by an impairment of synaptic transmission, at least in earlier stages, rather than neuronal apoptosis. 
In these experiments, the analysis is focused on particular signals that are known to be of synaptic origin, which can either be accomplished by electrical stimulation via the integrated electrodes of MEAs or via usage of chemicals that antagonize synaptic potentials. Ahuja et al. exposed hippocampal cultures integrated into MEA chips to either theta-burst (TBS, 10 trains of 4 pulses with a total duration of $2 \mathrm{~s}$ ) or high-frequency stimulation (HFS, two 1-s trains), both using a frequency of $100 \mathrm{~Hz}$, to induce initial LTP (Ahuja et al. 2007), which was calculated as the average of evoked EPSPs in a period of $5 \mathrm{~min}$. Following long-term exposure $(24 \mathrm{~h})$ to aggregated $A \beta_{42}(1 \mu \mathrm{M})$, they found no changes in spontaneous EPSPs, while the ability of hippocampal tissue to induce LTP was decreased globally. The same was found in experiments with mice of an AD model overexpressing $A \beta_{42}$ and tau in combination (Chong et al. 2011).

Using a different approach in confirming the synaptic origin of measured signals, further studies not only showed declining of synaptic activity in hippocampal neurons following application of different concentrations of oligomeric $A \beta_{42}(10 \mathrm{nM}, 1 \mu \mathrm{M}$ and $5 \mu \mathrm{M})$, but also that activity returns to baseline after $120 \mathrm{~h}$, indicating metabolic processing of oligomers (internalization and/or proteolysis) by hippocampal tissue (Lee et al. 2013). The synaptic origin of the signals was not ascertained recording them after electrical stimulation, but by inhibiting excitatory and inhibitory synaptic potentials using a mixture of certain antagonists, as described earlier (Serra et al. 2010). The addition of iron to hippocampal cultures seems to synergistically enhance the inhibition of synaptic signaling to an extent at which even subcytotoxic levels of $A \beta$ suffice, as shown in a consecutive study (Shea 2014). Moreover, this effect could be reversed by adding zinc, which prevented frequency of synaptic signals from declining. Based on earlier studies on levels of iron and zinc in spinal fluid of individuals with $\mathrm{AD}$ versus healthy controls, the authors propose that the interaction of certain metals with physiological species of $A \beta$ might be an initiation point of the disease, which so far has not been taken into consideration due to the relatively subtle impact on synaptic signaling, a factor that is certainly more difficult to examine in humans.

\section{Conclusions}

Overall, the studies described here corroborate a stateof-the-art hypothesis on $\mathrm{A} \beta$ inducing neurotoxicity on an electrophysiological level using MEA technology. This technique opens a large field of experimental possibilities, ranging from pharmacological studies to the investigation of communication in neural networks, including whole brain slices and single cell type cultures. Hence, MEA technology is a highly suitable tool for the examination of neurodegenerative disorders such as $\mathrm{AD}$, enabling the analysis of deleterious influences of diseaserelated peptides onto neural tissue not only on the level of the whole network, but also making it possible to examine the effects onto synaptic currents. This review focused on experiments studying the impact of $A \beta$ onto hippocampal neurons in vitro, while current and future studies will also be based on multielectrode array measurements in vivo, using electrodes implanted into cerebral tissue, moving the scope even closer to the conditions that cause neurodegeneration and how the typical symptoms of these disorders emerge.

\section{Abbreviations}

AD: Alzheimer's disease; A $\beta$ : amyloid- $\beta$; EAP: extracellular action potential; EPSP: excitatory post-synaptic potential; HFS: high-frequency stimulation; IAP: intracellular action potential; LFP: local field potential;

MEA: multielectrode array; ms: milliseconds; nAChR: nicotininc Acetylcholine receptor; TBS: theta-burst stimulation

\section{Acknowledgements}

Not applicable.

\section{Authors' contributions}

StS and MG conceptualized and designed the study. AC contributed to many discussions at different stages of manuscript development and supported literature review. All authors contributed to drafts of the manuscript. All authors read and approved the final submitted manuscript.

Funding

Not applicable. Open Access funding enabled and organized by Projekt DEAL.

Availability of data and materials

Not applicable.

\section{Declarations}

Ethics approval and consent to participate

Not applicable.

Consent for publication

Not applicable.

\section{Competing interests}

The authors declare that they have no competing interests.

Received: 16 August 2021 Accepted: 5 October 2021

Published online: 28 October 2021

\section{References}

Ahuja TK, Mielke JG, Comas T, et al. Hippocampal slice cultures integrated with multi-electrode arrays: A model for study of long-term drug effects on synaptic activity. Drug Dev Res. 2007;68:84-93. https://doi.org/10.1002/ddr.2 0170.

Buccino AP, Kordovan M, Ness TV, et al. Combining biophysical modeling and deep learning for multielectrode array neuron localization and classification. J Neurophysiol. 2018;120:1212-32. https://doi.org/10.1152/jn.00210.2018.

Buzsáki G, Anastassiou CA, Koch C. The origin of extracellular fields and currentsEEG, ECoG, LFP and spikes. Nat Rev Neurosci. 2012;13:407-20. https://doi. org/10.1038/nrn3241.

Chen QS, Kagan BL, Hirakura Y, Xie CW. Impairment of hippocampal long-term potentiation by Alzheimer amyloid $\beta$-peptides. J Neurosci Res. 2000;60:65-72. https://doi.org/10.1002/(SICl) 1097-4547(20000401)60:1<65::AID-JNR7>3.0.CO;2-Q

Chong SA, Benilova I, Shaban H, et al. Synaptic dysfunction in hippocampus of transgenic mouse models of Alzheimer's disease: A multi-electrode array study. Neurobiol Dis. 2011;44:284-91. https://doi.org/10.1016/j.nbd.2011.07.006.

Decker D, Hempelmann R, Natter H, et al. 3D Nanostructured Multielectrode Arrays: Fabrication, Electrochemical Characterization, and Evaluation of Cell- 
Electrode Adhesion. Adv Mater Technol. 2019;4:1-10. https://doi.org/10.1 002/admt.201800436

Delgado Ruz I, Schultz SR. Localising and classifying neurons from high density MEA recordings. J Neurosci Methods. 2014;233:115-28. https://doi.org/10.101 6/j.jneumeth.2014.05.037.

Glenner G, Wong WC. Alzheimer's disease and Down's syndrome: sharing of a unique cerebrovascular amyloid fibril protein. Biochem Biophys Res Commun. 1984;122:1131-5.

Gopal KV, Wu C, Moore EJ, Gross GW. Assessment of Styrene Oxide Neurotoxicity Using In Vitro Auditory Cortex Networks. ISRN Otolaryngol. 2011;2011:1-8. https://doi.org/10.5402/2011/204804.

Gramowski A, Jügelt K, Stüwe S, et al. Functional screening of traditional antidepressants with primary cortical neuronal networks grown on multielectrode neurochips. Eur J Neurosci. 2006;24:455-65. https://doi.org/1 0.1111/j.1460-9568.2006.04892.x

Gramowski A, Jügelt K, Schröder OHU, et al. Acute functional neurotoxicity of lanthanum(III) in primary cortical networks. Toxicol Sci. 2011;120:173-83. https://doi.org/10.1093/toxsci/kfq385.

Grutzendler J, Helmin K, Tsai J, Gan WB. Various dendritic abnormalities are associated with fibrillar amyloid deposits in Alzheimer's disease. Ann N Y Acad Sci. 2007;1097:30-9. https://doi.org/10.1196/annals.1379.003.

Hales CM, Zeller-Townson R, Newman JP, et al. Stimulus-evoked high frequency oscillations are present in neuronal networks on microelectrode arrays. Front Neural Circuits. 2012;6:1-10. https://doi.org/10.3389/fncir.2012.00029.

Henderson BW, Greathouse KM, Ramdas R, et al. Pharmacologic inhibition of LIMK1 provides dendritic spine resilience against $\beta$-amyloid. Sci Signal. 2019; 12:eaaw9318. https://doi.org/10.1126/scisignal.aaw9318.

Herreras O. Local field potentials: Myths and misunderstandings. Front Neural Circuits. 2016;10:1-16. https://doi.org/10.3389/fncir.2016.00101.

Hofmann F, Bading H. Long term recordings with microelectrode arrays: Studies of transcription-dependent neuronal plasticity and axonal regeneration. J Physiol. 2006;99:125-32. https://doi.org/10.1016/j.jphysparis.2005.12.005.

Hoppe JB, Haag M, Whalley BJ, et al. Curcumin protects organotypic hippocampal slice cultures from A 1 1-42-induced synaptic toxicity. Toxico Vitr. 2013;27:2325-30. https://doi.org/10.1016/j.tiv.2013.10.002.

Johnston D, Magee JC, Colbert CM, Christie BR. Active properties of neurona dendrites. Annu Rev Neurosci. 1996;19:165-86. https://doi.org/10.1146/a nnurev.ne.19.030196.001121.

Jones IL, Livi P, Lewandowska MK, et al. The potential of microelectrode arrays and microelectronics for biomedical research and diagnostics. Anal Bioanal Chem. 2011;399:2313-29. https://doi.org/10.1007/s00216-010-3 968-1.

Killian NJ, Vernekar VN, Potter SM, Vukasinovic J. A device for long-term perfusion, imaging, and electrical interfacing of brain tissue in vitro. Front Neurosci. 2016;10:1-14. https://doi.org/10.3389/fnins.2016.00135.

Koffie RM, Hyman BT, Spires-Jones TL. Alzheimer's disease: Synapses gone cold. Mol Neurodegener. 2011;6:63. https://doi.org/10.1186/1750-1326-6-63.

Lee S, Zemianek J, Shea TB. Rapid, reversible impairment of synaptic signaling in cultured cortical neurons by exogenously-applied amyloid- $\beta$. J Alzheimer's Dis. 2013;35:395-402. https://doi.org/10.3233/JAD-122452.

Lewicki MS. A review of methods for spike sorting: The detection and classification of neural action potentials. Netw Comput Neural Syst. 1998;9: R53-78. https://doi.org/10.1088/0954-898X_9_4_001.

Minkeviciene R, Rheims S, Dobszay MB, et al. Amyloid $\beta$-induced neuronal hyperexcitability triggers progressive epilepsy. J Neurosci. 2009;29:3453-62. https://doi.org/10.1523/JNEUROSCI.5215-08.2009.

Morefield SI, Keefer EW, Chapman KD, Gross GW. Drug evaluations using neuronal networks cultured on microelectrode arrays. Biosens Bioelectron. 2000;15:383-96. https://doi.org/10.1016/S0956-5663(00)00095-6.

Ness TV, Chintaluri C, Potworowski J, et al. Modelling and Analysis of Electrical Potentials Recorded in Microelectrode Arrays (MEAs). Neuroinformatics. 2015; 13:403-26. https://doi.org/10.1007/s12021-015-9265-6.

Nowduri B, Schulte S, Decker D, et al. Biomimetic Nanostructures Fabricated by Nanoimprint Lithography for Improved Cell-Coupling. Adv Funct Mater. 2020; 30:1-10. https://doi.org/10.1002/adfm.202004227.

Obien MEJ, Deligkaris K, Bullmann T, et al. Revealing neuronal function through microelectrode array recordings. Front Neurosci. 2015;9:423. https://doi.org/1 0.3389/fnins.2014.00423.

Patriarchi T, Cho JR, Merten K, et al. Ultrafast neuronal imaging of dopamine dynamics with designed genetically encoded sensors. Science. 2018:360:122. https://doi.org/10.1126/science.aat4422.Ultrafast.
Potter SM, Demarse TB. A new approach to neural cell culture for long-term studies. J Neurosci Methods. 2001:110:17-24.

Quiroga R, Panzeri S, Anastassiou C, et al. Biophysics of Extracellular Spikes. Princ Neural Coding. 2013;15-36. https://doi.org/10.1201/b14756-4.

Sabatini BL, Tian L. Primer Imaging Neurotransmitter and Neuromodulator Dynamics In Vivo with Genetically Encoded Indicators. Neuron. 2020;108:1732. https://doi.org/10.1016/j.neuron.2020.09.036.

Saif-Ur-Rehman M, Lienk mper R, Parpaley Y, et al. SpikeDeeptector: A deeplearning based method for detection of neural spiking activity. J Neural Eng. 2019;16:056003. https://doi.org/10.1088/1741-2552/ab1e63.

Serra M, Guaraldi M, Shea TB. Inhibitory neurons modulate spontaneous signaling in cultured cortical neurons: Density-dependent regulation of excitatory neuronal signaling. Phys Biol. 2010;7:026009. https://doi.org/10.1088/1478-3 975/7/2/026009.

Shahaf G, Marom S. Learning in Networks of Cortical Neurons. J Neurosci. 2001; 21:8782-8

Shea TB. Synergistic inhibition of synaptic signaling in cortical cultures by subcytotoxic levels of oligomerized amyloid- $\beta$ and iron: alleviation by zinc. J Alzheimers Dis. 2014;41:365-9. https://doi.org/10.3233/JAD-132696.

Šišková Z, Justus D, Kaneko H, et al. Dendritic structural degeneration is functionally linked to cellular hyperexcitability in a mouse model of alzheimer's disease. Neuron. 2014:84:1023-33. https://doi.org/10.1016/j.neuron.2014.10.024.

Spira ME, Hai A. Multi-electrode array technologies for neuroscience and cardiology. Nat Nanotechnol. 2013;8:83-94. https://doi.org/10.1038/nnano.2012.265.

Spruston N. Pyramidal neurons: Dendritic structure and synaptic integration. Nat Rev Neurosci. 2008:9:206-21. https://doi.org/10.1038/nrn2286.

St George-Hyslop PH, Petit A. Molecular biology and genetics of Alzheimer's disease. Comptes Rendus - Biol. 2005;328:119-30. https://doi.org/10.1016/j. crvi.2004.10.013.

Tavanaei A, Ghodrati M, Kheradpisheh SR, et al. Deep learning in spiking neural networks. Neural Netw. 2019;111:47-63. https:/doi.org/10.1016/j.neunet.2018.12.002.

Townsend M, Shankar GM, Mehta T, et al. Effects of secreted oligomers of amyloid B-protein on hippocampal synaptic plasticity: A potent role for trimers. J Physiol. 2006;572:477-92. https://doi.org/10.1113/jphysiol.2005.103754.

Varghese K, Molnar P, Das M, et al. A new target for amyloid beta toxicity validated by standard and high-throughput electrophysiology. PLoS One. 2010;5:e8643. https://doi.org/10.1371/journal.pone.0008643.

Wagner FB, Eskandar EN, Cosgrove GR, et al. Microscale spatiotemporal dynamics during neocortical propagation of human focal seizures. Neuroimage. 2015; 122:114-30. https://doi.org/10.1016/j.neuroimage.2015.08.019.

Walker LC. Aß P. Free Neuropathol. 2020;1:1-72. https://doi.org/10.17879/ freeneuropathology-2020-3025.A.

Wang HY, Lee DHS, Davis CB, Shank RP. Amyloid peptide A $1-42$ binds selectively and with picomolar affinity to a7 nicotinic acetylcholine receptors. J Neurochem. 2000;75: 1155-61. https://doi.org/10.1046/j.1471-4159.2000.0751155.x.

Wang HW, Pasternak JF, Kuo H, et al. Soluble oligomers of $\beta$ amyloid (1-42) inhibit long-term potentiation but not long-term depression in rat dentate gyrus. Brain Res. 2002;924:133-40. https://doi.org/10.1016/S0006-8993(01)03058-X

Wood JG, Mirra SS, Pollock NJ, Binder LI. Neurofibrillary tangles of Alzheimer disease share antigenic determinants with the axonal microtubule-associated protein tau ( (). Proc Natl Acad Sci U S A. 1986;83:4040-3. https://doi.org/10.1 073/pnas.83.11.4040.

Wood C, Williams C, Waldron GJ. Patch clamping by numbers. Drug Discov Today. 2004;9:434-41. https://doi.org/10.1016/S1359-6446(04)03064-8.

Yang F, Lim GP, Begum AN, et al. Curcumin inhibits formation of amyloid $\beta$ oligomers and fibrils, binds plaques, and reduces amyloid in vivo. J Biol Chem. 2005;280:5892-901. https://doi.org/10.1074/jbc.M404751200.

Zheng $Y$, Tian C, Dong L, et al. Effect of arsenic-containing hydrocarbon on the long-term potentiation at Schaffer Collateral-CA1 synapses from infantile male rat. Neurotoxicology. 2021;84:198-207. https:/doi.org/10.1016/..neuro.2021.04.002.

H. Alzoubi K, A. Alhaider I, T. Tran T, et al. Impaired Neural Transmission and Synaptic Plasticity in Superior Cervical Ganglia from $\beta$-Amyloid Rat Model of Alzheimers Disease. Curr Alzheimer Res. 2011:8:377-384. https://doi.org/10. 2174/156720511795745311.

\section{Publisher's Note}

Springer Nature remains neutral with regard to jurisdictional claims in published maps and institutional affiliations. 